\title{
OSCILLATION OF NEUTRAL DIFFERENTIAL EQUATIONS WITH PERIODIC COEFFICIENTS
}

\author{
QINGGUANG HUANG AND SHAOZHU CHEN
}

(Communicated by Kenneth R. Meyer)

\begin{abstract}
We establish a necessary and sufficient condition for the oscillation of all solutions to the neutral differential equation

$$
[x(t)-p(x)(t-r)]^{\prime}+\sum_{i=1}^{n} q_{i}(t) x(t-i r)=0,
$$

where $0 \leq p \leq 1, r>0$ are constants and $q_{i}(t) \geq 0, i=1, \ldots, n$, are continuous $r$-periodic functions.
\end{abstract}

\section{INTRODUCTION}

We are concerned with the neutral differential equation

$$
[x(t)-p x(t-r)]^{\prime}+\sum_{i=1}^{n} q_{i}(t) x(t-i r)=0, \quad{ }^{\prime}=\frac{d}{d t},
$$

where $0 \leq p \leq 1, r>0$ are constants and $q_{i}(t) \geq 0, i=1, \ldots, n$, are continuous, $r$-periodic functions on $\mathbf{R}$.

By a solution of equation (1) on $[T-n r, \infty), T \in \mathbf{R}$, we mean a continuous function $x(t)$ on $[T-n r, \infty)$ for which $x(t)-p x(t-r)$ is continuously differentiable and (1) is satisfied for $t \geq T$. A solution $x(t)$ of equation (1) is called oscillatory if it has arbitrarily large zeros. Otherwise, it is called nonoscillatory. If every solution of (1) is oscillatory, then we will call equation (1) oscillatory, and otherwise nonoscillatory.

Let $Q_{i}=\int_{0}^{r} q_{i}(s) d s, i=1, \ldots, n$, and assume $Q_{n}>0$. We will call the following equation

$$
\lambda r\left(1-p e^{-\lambda r}\right)+\sum_{i=1}^{n} Q_{i} e^{-i \lambda r}=0
$$

the characteristic equation of (1). This terminology comes from the autonomous case where each function $q_{i}(t)$ is identically equal to a constant, $i=1, \ldots, n$.

Received by the editors August 14, 1989.

1980 Mathematics Subject Classification (1985 Revision). Primary 34K15; Secondary 34K25. tions.

Key words and phrases. Oscillation, neutral equations, periodic coefficients, characteristic equa- 
There has recently been extensive literature about oscillations of both autonomous and nonautonomous neutral differential equations (see [1-4], [6], [8] and the references cited therein). For oscillations in autonomous cases, many authors have in particular obtained necessary and sufficient conditions involving the nonexistence of real characteristic roots [1], [4], [6], [8]. It is natural to ask whether or, more precisely, to what extent it is possible to do the same for nonautonomous neutral equations. In this paper we will answer the question partially, that is, we will prove that equation (1) is oscillatory if and only if equation (2) has no real roots. To the best of the authors' knowledge, this is the first paper that characterizes the oscillation of neutral equations with periodic coefficients. The referee has informed the authors that similar results were found independently by G. Ladas, Ch. G. Philos, and Y. G. Sficas. For delay differential equations with periodic coefficients with or without sign restrictions on the coefficients we refer to [7] or [5], respectively.

\section{THE MAIN RESULT}

The following two lemmas will be needed. For the proof of the first one we refer to [2].

Lemma 1. If $x(t)$ is a solution of equation (1), then $v(t)=x(t)-p x(t-r)$ is also a solution of (1). Moreover, if $x(t)$ is eventually positive, then $v(t)$ and $-v^{\prime}(t)$ are also eventually positive.

Lemma 2. If $x(t)$ is an eventually positive solution of equation (1), then there exists a constant a such that

$$
\limsup _{t \rightarrow \infty} x(t) e^{a t}>0 .
$$

Proof. By Lemma 1, there is a $T \in \mathbf{R}$ such that $x(t)>v(t), v^{\prime}(t)<0$ for $t \geq T$. From (1), we have

$$
0=v^{\prime}(t)+\sum_{i=1}^{n} q_{i}(t) x(t-i r) \geq v^{\prime}(t)+q_{n}(t) v(t-n r), \quad t \geq T+n r .
$$

Since $q_{n}(t)$ is $r$-periodic and $Q_{n}>0$, there must be a $b \in(0, r)$ such that

$$
\int_{t-b}^{t} q_{n}(s) d s \geq k=\frac{1}{2} Q_{n} \text { for all } t .
$$

Integrating (4) from $t$ to $t+b$ yields

$$
\begin{aligned}
& v(t+b)-v(t)+v(t+b-r) \int_{t}^{t+b} q_{n}(s) d s \\
& \quad \leq v(t+b)-v(t)+\int_{t}^{t+b} q_{n}(s) v(s-n r) d s \leq 0, \quad t \geq T+n r .
\end{aligned}
$$

From (5) and (6) we have

$$
v(t) \geq k v(t-(r-b)), \quad t \geq T+n r
$$


and can then prove without difficulty that there is an $M>0$ such that

$$
v(t) \geq M \exp \left(\frac{t}{r-b} \ln k\right) .
$$

Thus (3) holds for $a=(-\ln k) /(r-b)$ since $x(t)>v(t)$. The proof of Lemma 2 is complete.

The following theorem is our main result.

Theorem. Equation (1) is oscillatory if and only if its characteristic equation (2) has no real roots.

Proof. The necessity of the theorem is obvious, for if (2) has a real root $\lambda$, then the function

$$
x(t)=\exp \left[-\frac{1}{1-p e^{-\lambda r}} \sum_{i=1}^{n} \int_{0}^{t} q_{i}(s) e^{-i \lambda r} d s\right]
$$

is a nonoscillatory solution of (1).

To prove the sufficiency, we assume (1) is nonoscillatory. Without loss of generality, let $x(t)$ be an eventually positive solution of (1). Let $v(t)=x(t)-$ $p x(t-r)$. In view of Lemma $1, v(t)$ is a solution of $(1), v(t)>0$, and $v^{\prime}(t)<0$ for all large $t$, say, $t \geq T \geq 0$.

Let $u \in C\left([T, \infty), \mathbf{R}_{+}\right)$be such that $v(t)=v(T) \exp \left(-\int_{T}^{t} u(s) d s\right)$. Then $u(t)$ satisfies

$$
\begin{aligned}
u(t)= & p u(t-r) \exp \int_{t-r}^{t} u(s) d s \\
& +\sum_{i=1}^{n} q_{i}(t) \exp \int_{t-i r}^{t} u(s) d s, \quad t \geq t_{1}=T+n r
\end{aligned}
$$

Set $z_{0}(t)=0$ and for $k=0,1,2, \ldots$ set

$$
\begin{aligned}
z_{k+1}(t)= & p z_{k}(t-r) \exp \int_{t-r}^{t} z_{k}(s) d s \\
& +\sum_{i=1}^{n} q_{i}(t) \exp \int_{t-i r}^{t} z_{k}(s) d s, \quad t \geq t_{1}, \\
= & z_{k+1}\left(t_{1}\right), \quad T \leq t<t_{1} .
\end{aligned}
$$

Then, it follows by induction that

$$
z_{k}(t) \leq z_{k+1}(t), \quad t \geq T
$$

and

$$
z_{k}(t) \leq u(t), \quad t \geq t_{k}=T+k n r, \quad k=0,1,2, \ldots .
$$

For $i=1, \ldots, n$, set $\lambda_{0}^{(i)}=0$ and set

$$
\lambda_{k+1}^{(i)}=p \lambda_{k}^{(i)} \exp \left(\sum_{j=1}^{n} Q_{j} \lambda_{k}^{(j)}\right)+\exp \left(i \sum_{j=1}^{n} Q_{j} \lambda_{k}^{(j)}\right), \quad k=0,1,2, \ldots
$$


From (8) and (11), it then follows by induction (here we omit the tedious computation) that

$$
z_{k}(t)=\sum_{i=1}^{n} q_{i}(t) \lambda_{k}^{(i)}, \quad t \geq T, k=0,1,2, \ldots
$$

Inductively, we also obtain from (11) that

$$
\lambda_{k}^{(i)} \leq \lambda_{k+1}^{(i)}, \quad i=1, \ldots, n, k=0,1, \ldots
$$

and

$$
\lambda_{k}^{(i)} \leq \lambda_{k}^{(i+1)}, \quad k=0,1, \ldots, i=1, \ldots, n-1 .
$$

We claim that $\lambda_{k}^{(n)}$ is bounded above as $k \rightarrow \infty$. If this is not true, then for any $M>0$, there is a $k>1$ such that $\lambda_{k}^{(n)} Q_{n}>M r$. Hence, from (10) and (12) we have for $t>t_{k}+r$,

$$
\begin{aligned}
v(t) & =v(T) \exp \left(-\int_{T}^{t} u(s) d s\right)=v(T) \exp \left(-\int_{T}^{t_{k}} u(s) d s-\int_{t_{k}}^{t} u(s) d s\right) \\
& \leq B \exp \left(-\int_{t_{k}}^{t} z_{k}(s) d s\right) \leq B \exp \left(-\lambda_{k}^{(n)} \int_{t_{k}}^{t} q_{n}(s) d s\right) \\
& \leq B \exp \left(-\lambda_{k}^{(n)} Q_{n}\left[\left(t-t_{k}\right) / r\right]\right) \leq B \exp \left(-M\left(t-t_{k}-r\right)\right),
\end{aligned}
$$

where $B>0$ is a constant and $\left[\left(t-t_{k}\right) / r\right]$ is the greatest integer in $\left(t-t_{k}\right) / r$. This contradicts Lemma 2. Thus, $\lambda_{k}^{(n)}$ is bounded above as $k \rightarrow \infty$. Then (13) and (14) allow us to assure that $\lambda_{k}^{(i)}$ tends to a finite limit $\alpha_{i}$ as $k \rightarrow \infty$ for each $i=1, \ldots, n$. Letting $k \rightarrow \infty$ in (11) produces

$$
\alpha_{i}=p \alpha_{i} e^{\mu}+e^{i \mu}, \quad i=1, \ldots, n,
$$

where $\mu=\sum_{i=1}^{n} Q_{i} \alpha_{i}$. Multiplying (15) by $Q_{i}$ and then summing up over $i=1, \ldots, n$, we get

$$
\mu=p \mu e^{\mu}+\sum_{i=1}^{n} Q_{i} e^{i \mu}
$$

The equality (16) implies that $-\mu / r$ is a real root of (2). The contradiction completes the proof.

\section{REFERENCES}

1. S. Chen and Q. Huang, Necessary and sufficient conditions for the oscillation of solutions to systems of neutral functional differential equations, Funkcial. Ekvac. (to appear).

2. M. K. Grammatikopoulos, G. Ladas, and Y. G. Sficas, Oscillation and asymptotic behavior of neutral equations with variable coefficients, Rad. Mat. 2 (1986), 279-303.

3. E. A. Grove, M.R.S. Kulenović, and G. Ladas, Sufficient conditions for oscillation and nonoscillation of neutral equations, J. Differential Equations 68 (1987), 373-382.

4. E. A. Grove, G. Ladas, and A. Meimaridou, A necessary and sufficient condition for the oscillation of neutral equations, J. Math. Anal. Appl. 126 (1987), 34i-354. 
5. Q. Huang, Necessary and sufficient conditions for the oscillation of a class of differential difference equations with sign-variable periodic coefficients, Kexue Tongbao 33 (1988), 156.

6. M. R. S. Kulenović, G. Ladas, and A. Meimaridou, Necessary and sufficient conditions for oscillations of neutral differential equations, J. Austral. Math. Soc. Ser. B 28 (1987), 362-375.

7. G. Ladas, Y. G. Sficas, and I. P. Stavroulakis, Nonoscillatory functional differential equations, Pacific J. Math. 115 (1984), 391-398.

8. Y. G. Sficas and I. D. Stavroulakis, Necessary and sufficient conditions for oscillations of neutral differential equations, J. Math. Anal. Appl. 123 (1987), 494-507.

Department of Mathematics, Shandong University, Jinan, Shandong, 250100, People's REPUBLIC OF CHINA 